\title{
Trends in resistant Enterobacteriaceae and Acinetobacter species in hospitalized patients in the United States: 2013-2017
}

\author{
Vikas Gupta ${ }^{1 *}$, Gang Ye ${ }^{1}$, Melanie Olesky², Kenneth Lawrence ${ }^{2}$, John Murray ${ }^{1}$ and Kalvin Yu
}

\begin{abstract}
Background: Trends in antimicrobial resistance help inform infection control efforts. We examined trends in resistance for Enterobacteriaceae and Acinetobacter spp. from 2013 to 2017 in hospitalized US patients.

Methods: We analyzed antimicrobial susceptibility of non-duplicate isolates in hospitalized patients (not limited to hospital-acquired infections) in the US BD Insights Research Database. Resistance profiles of interest were extendedspectrum beta-lactamase (ESBL)-producing, multidrug resistant (MDR), and carbapenem-nonsusceptible (Carb-NS) phenotypes of Enterobacteriaceae, and MDR and Carb-NS Acinetobacter spp. Time series models were used to evaluate the patterns of resistance trends in rate per 100 hospital admissions and proportion per isolates tested.

Results: More than 1 million Enterobacteriaceae isolates were obtained from 411 hospitals; 12.05\% were ESBL, 1.21\% Carb-NS, and 7.08\% MDR. Urine was the most common source. For Acinetobacter spp. $(n=19,325), 37.48 \%$ were Carb-NS, 47.66\% were MDR, and the most common source was skin/wound cultures. Trend analyses showed that the rates of ESBL and Carb-NS Enterobacteriaceae per 100 hospital admissions increased significantly between 2013 and 2017. Rates of MDR Enterobacteriaceae and Carb-NS and MDR Acinetobacter spp. decreased during this time period. Trends in proportions of resistant isolates generally mirrored trends in rates per 100 hospital admissions. MDR Enterobacteriaceae and Carb-NS and MDR Acinetobacter spp. were more common in winter than summer.
\end{abstract}

Conclusions: In this large-scale study of patients in US hospitals, rates of ESBL and Carb-NS Enterobacteriaceae per 100 hospital admissions increased between 2013 and 2017. MDR Enterobacteriaceae and MDR and Carb-NS Acinetobacter spp. isolates decreased over this period. These data support continuing infection control and stewardship efforts and the development of new therapeutic options.

Keywords: Antimicrobial resistance, Acinetobacter, Enterobacteriaceae, United States, Carbapenems, Extendedspectrum beta-lactamases, Multidrug resistance

\section{Background}

Antibiotic-resistant Gram-negative bacteria have been recognized as a fundamental risk to patient health on both national and global levels [1-3]. Enterobacteriaceae, which account for a significant proportion of infections in hospitalized patients in the US [4, 5], are of particular concern. Carbapenem-resistant Enterobacteriaceae (CRE) have limited treatment options and thus pose a significant clinical dilemma, but extended-spectrum beta-lactamase

\footnotetext{
* Correspondence: vikas.gupta@bd.com

'Becton, Dickinson and Company, 1 Becton Drive, Franklin Lakes, NJ, USA

${ }^{2}$ Tetraphase Pharmaceuticals, Watertown, MA, USA
}

(ESBL)-producing Enterobacteriaceae and multidrug-resistant (MDR) Enterobacteriaceae are also difficult to treat and occur at a higher prevalence, therefore endangering a greater number of patients [6]. The Centers for Disease Control and Prevention (CDC) categorizes CRE and ESBL-producing Enterobacteriaceae as urgent and serious threats, respectively, and the World Health Organization (WHO) considers them a critical priority for drug development [1-3]. Carbapenem-resistant and MDR Acinetobacter are also featured on the CDC and WHO lists of dangerous pathogens [1-3]. Although Acinetobacter infections are relatively rare,

(c) The Author(s). 2019 Open Access This article is distributed under the terms of the Creative Commons Attribution 4.0 International License (http://creativecommons.org/licenses/by/4.0/), which permits unrestricted use, distribution, and 
this pathogen is difficult to treat due to high rates of antibiotic resistance and paucity of options [7].

Trends in resistance patterns provide important insights into emerging pathogens as well as inform public health, infection control, and antimicrobial stewardship approaches. Although comprehensive data on hospitalacquired infections, including catheter-associated urinary tract infections (UTIs) and central-line associated bloodstream infections (BSIs), are available from the US National Healthcare Safety Network (NHSN) [6, 8], national comparative data for antimicrobial-resistant Gram-negative pathogens in the complete population of hospitalized patients are not as easily accessed. The objective of this study was to examine trends in resistance in selected Gram-negative pathogens collected from US hospitals from 2013 through 2017 based on rates of resistance per 100 hospital admissions and proportions of resistant isolates.

\section{Methods}

\section{Study design and participating hospitals}

The study reported here was a retrospective longitudinal analysis of antimicrobial susceptibility of all specified non-duplicate (first isolate of a species within 30 days) Enterobacteriaceae and Acinetobacter species (spp.) isolates from hospitalized patients from the first quarter (Q1) of 2013 through the last quarter (Q4) of 2017. Isolates from respiratory, blood, urine, skin/wound, intra-abdominal, and other culture sources were included. Microbiology results likely associated with surveillance cultures (eg, nasal or rectal swabs) and environmental cultures were excluded from this analysis by previously described methodology that uses source, time of collection, pathogen type, and number of pathogens in a culture to flag likely contaminated samples [9]. Reporting institutions consisted of US hospitals in the BD Insights Research Database (Becton, Dickinson and Company, Franklin Lakes, NJ). The clinical research database (formerly referred to as the CareFusion Clinical Research Database) has been described previously [9-11]. This database provides geographical representation across the US; both small and large hospitals in rural and urban areas are included. Susceptibility results and pathogen identification were based on facility reports from hospitals in the database. There was no central laboratory or standardization of breakpoints or testing methods.

Our study evaluated antimicrobial susceptibility in five groups of Gram-negative bacteria using the following definitions as previously described [12, 13]:

1. ESBL Enterobacteriaceae: Escherichia coli, Klebsiella oxytoca, Klebsiella pneumoniae, and Proteus mirabilis isolates confirmed as ESBL positive by commercial laboratory panels OR with intermediate susceptibility or resistance to cefepime, ceftazidime, cefotaxime, or ceftriaxone.

2. MDR Enterobacteriaceae: Citrobacter freundi, Enterobacter aerogenes, Enterobacter cloacae, E. coli, K. oxytoca, K. pneumoniae, Morganella morganii, $P$. mirabilis, and Serratia marcescens isolates with intermediate susceptibility or resistance to at least one drug in three of the five following classes: aminoglycosides, carbapenems, extended-spectrum cephalosporins, fluoroquinolones, and piperacillin or piperacillin/tazobactam (see Additional file 1 for specific drugs) $[6,14]$.

3. Carbapenem-nonsusceptible (Carb-NS) Enterobacteriaceae: C. freundi, E. aerogenes, $E$. cloacae, E. coli, K. oxytoca, K. pneumoniae, M. morganii, P. mirabilis, and S. marcescens isolates with intermediate susceptibility or resistance to imipenem (excluded for $M$. morganii and $P$. mirabilis), doripenem, ertapenem, or meropenem, The inclusion of both intermediate susceptibility and resistance is consistent with the CRE definition used by the $\mathrm{CDC}$ in their facility guidance document (2012 CRE toolkit), although the 2012 definition did not include ertapenem as a carbapenem [15]. It should be noted that the CDC CRE definition was revised in 2015 to include only Enterobacteriaceae resistant (rather than nonsusceptible) to any carbapenem (doripenem, meropenem, imipenem, or ertapenem) or documented to produce carbapenemase [15].

4. MDR Acinetobacter spp.: Acinetobacter baumannii/Acinetobacter haemolyticus (henceforth referred to as Acinetobacter spp.) isolates with intermediate susceptibility or resistance to at least one drug in three of the six following classes: ampicillin/sulbactam, aminoglycosides, carbapenems, extendedspectrum cephalosporins, fluoroquinolones, and piperacillin or piperacillin/tazobactam (see Additional file 1).

5. Carb-NS Acinetobacter spp.: A. baumannii/A. haemolyticus isolates with intermediate susceptibility or resistance to imipenem, doripenem, or meropenem.

\section{Outcomes}

The outcomes assessed were the rate of bacterial resistance or non-susceptibility, as defined above, per 100 hospital admissions and the proportion of resistant isolates (number of resistant isolates divided by number of nonduplicate isolates tested) for each year-quarter from Q1 2013 through Q4 2017. Hospital admissions, which are frequently used as a denominator in reports of 
antimicrobial resistance [16, 17], were identified using available census, admission, discharge and transfer files, which were provided by each institution on a more than daily basis.

\section{Statistical analysis}

We employed a two-phase approach to analyze the data. As an exploratory phase, we performed a number of regression modeling analyses with hospital bed size, teaching or non-teaching status, urban or rural status, and geographic locations (regions) as covariates. For the proportion of resistant isolates, we used both logistic regression models and generalized estimating equations (GEE) to estimate resistance. To estimate the resistance rate per 100 admissions, we used a general linear mixed model (GLMM) via Poisson regression model with hospital as random effect. These regression models have the ability to assess effects as well as account for clustercorrelation of data. However, our observed data had a significant autocorrelation (verified via Durbin-Watson test), differencing-autocorrelation, and seasonal or cyclic changing patterns. Although GEE and GLMM can account for within-cluster correlation to some degree (depending on appropriate choice of variance-covariance structures), these methods lack the ability to handle differencing-autocorrelation, seasonality, and periodicity. Therefore, in our second phase of analysis, we decided to use a time series analysis method to fit data. Time series modeling or smoothing methods employ more recent data information (compared with older data) in prediction and estimation of parameters and variances.

In the time series analysis phase, we found that our series data were not stationary or differencing-stationary and thus decided not to use the more popular autoregressive integrated moving average (ARIMA) models. We instead chose the unobserved component model (UCM) [18] to estimate trends in resistance data because our time series data showed certain non-Gaussian characteristics, structural breaks, and outliers. Time series models based on unobserved components are more flexible than regular time series models such as exponential smoothing and ARIMA models, and more effective in handling complex data [19]. Since more hospitals "entered" (contributed data to) the study over time, we postulated that the increasing number of hospitals could affect the estimates of outcome measures. Therefore, in each UCM, we created a time-varying variable, the quarterly number of hospitals contributing data, and modeled this variable as a random effect to see if it was a significant factor of the outcome measures. Our models showed that this time-varying variable had no significant impact.
All model results presented in this paper were generated using the UCM method. All statistical analyses were conducted using Statistical Analysis System (SAS) V9.4 (SAS Institute, Cary, NC) and SAS/ ETS 13.1. $P$ values $<0.05$ were considered statistically significant.

\section{Results}

A total of 411 hospitals provided data for this study (Table 1). About three-quarters of hospitals (76.6\%) were classified as urban, $70.6 \%$ were non-teaching hospitals, and the most common bed size was 100 to 300 (42.8\%). Geographically, the largest concentration of hospitals was in US Department of Health \& Human Services Region 4 (south central states; 23.6\%) and Region 5 (north central states; 23.4\%) (Table 1).

More than 1 million Enterobacteriaceae isolates were evaluated for ESBL (1,112,312 tested), Carb-NS (1,275, 311 tested), and MDR (1,275,311 tested). Over the 5year period, $12.05 \%$ Enterobacteriaceae isolates were identified as ESBL phenotype (hereafter referred to as ESBL Enterobacteriaceae), 1.21\% as Carb-NS, and 7.08\%

Table 1 Distribution of Hospitals Included in the Study

\begin{tabular}{|c|c|c|}
\hline Characteristic & $\mathrm{n}$ & $\%$ \\
\hline Overall & 411 & 100 \\
\hline \multicolumn{3}{|l|}{ HHS region (states) ${ }^{a}$} \\
\hline Region $1(\mathrm{CT}, \mathrm{ME}, \mathrm{MA}, \mathrm{NH}, \mathrm{RI}, \mathrm{VT})$ & 9 & 2.2 \\
\hline Region 2 (NJ, NY) & 45 & 11.0 \\
\hline Region 3 (DE, DC, MD, PA, VA, WV) & 18 & 4.4 \\
\hline Region 4 (AL, FL, GA, KY, MS, NC, SC, TN) & 97 & 23.6 \\
\hline Region 5 (IL, IN, MI, MN, OH, WI) & 96 & 23.4 \\
\hline Region 6 (AR, LA, NM, OK, TX) & 70 & 17.0 \\
\hline Region 7 (IA, KS, MO, NE) & 12 & 2.9 \\
\hline Region 8 (CO, MT, ND, SD, UT, WY) & 12 & 2.9 \\
\hline Region 9 (AZ, CA, HI, NV) & 34 & 8.3 \\
\hline Region 10 (AK, ID, OR, WA) & 18 & 4.4 \\
\hline \multicolumn{3}{|l|}{ Urban/Rural } \\
\hline Urban & 315 & 76.6 \\
\hline Rural & 96 & 23.4 \\
\hline \multicolumn{3}{|l|}{ Teaching status } \\
\hline Non-teaching & 290 & 70.6 \\
\hline Teaching & 121 & 29.4 \\
\hline \multicolumn{3}{|l|}{ Bed size } \\
\hline$<100$ & 109 & 26.5 \\
\hline $100-300$ & 176 & 42.8 \\
\hline$>300$ & 126 & 30.7 \\
\hline
\end{tabular}

Abbreviations: HHS US Department of Health \& Human Services ${ }^{a}$ US Territories were not included 
as MDR (Table 2). A total of 19,325 Acinetobacter spp. isolates were tested for Carb-NS and MDR. Of these isolates, $37.48 \%$ isolates were identified as Carb-NS and $47.66 \%$ as MDR.

For Enterobacteriaceae, urine cultures accounted for the majority of resistant isolates (66.66\% ESBL, 45.87\% Carb-NS, and $63.04 \%$ MDR), followed by skin/wound $(11.89,20.53$, and $13.12 \%$, respectively) (Table 2$)$. The highest rates of resistance were observed in respiratory cultures $(17.41,3.44$, and $10.23 \%$, and for ESBL, CarbNS, and MDR, respectively). For Acinetobacter spp., respiratory cultures were the most common source of Carb-NS (39.94\%) and MDR (39.29\%), and also had the highest proportion of Carb-NS and MDR isolates (45.00 and 56.29\%, respectively) (Table 2). Skin/wound was the second most common source of resistant Acinetobacter spp. (36.08\% for Carb-NS, 36.04\% for MDR).

Observational data for rates and proportions of antibiotic-resistant Enterobacteriaceae and Acinetobacter spp. from 2013 to 2017 are shown by quarter in Additional files 2 and 3, respectively.

\section{Trends in antibiotic-resistant Enterobacteriaceae}

Between 2013 and 2017, the rate of ESBL per 100 hospital admissions increased significantly in US hospitals. Statistical model-based assessment showed that the overall (linear) trend increased over time with an average slope of $0.0089 /$ quarter $(p<0.0001)$; no significant seasonal pattern was observed (Table 3 and Fig. 1a). Evaluations of the proportions of ESBL Enterobacteriaceae isolates (number of resistant isolates divided by number of isolates tested) found that ESBL Enterobacteriaceae increased from 10.1\% Q1 2013 to $12.6 \%$ in Q4 2017 (Additional file 2), with a similar pattern to that observed for rate per 100 hospital admissions (average slope of $0.151 \%$ /quarter; $p<0.0001$ ) (Table 3 and Fig. 1b). Seasonal variations were not significant.

Carb-NS Enterobacteriaceae also showed significant increases in rate per 100 hospital admissions between
2013 and 2017, although the trend was more gradual than observed with ESBL pathogens (slope of 0.0004/ quarter; $p=0.0047$ ) (Table 3 and Fig. 1c). Increases in the proportion of Carb-NS Enterobacteriaceae were not significant (1.2\% Q1 2013 to 1.3\% Q4 2017, $p=0.5331$ ) (Table 3 and Fig. 1d). No significant seasonal variations were observed for either rate or proportion of resistance in Carb-NS Enterobacteriaceae.

The rate of MDR Enterobacteriaceae per 100 hospital admissions showed an "up-down" nonlinear changing pattern, increasing prior to 2015 and decreasing during the most recent 3 -year time period (Table 3 and Fig. 1e). The overall trend between 2013 and 2017 was a slight but significant decrease in both rates per 100 admissions (slope of $-0.0022 ; p=0.0066$ ) and proportion of resistant pathogens (slope of $-0.0273 ; p=0.0176$ ) (Table 3 and Fig. 1e and f). The proportion of resistant isolates showed modest but significant seasonal variation, with higher rates in the winter period (Q1) compared with the summer period (Q3) (Table 3).

\section{Trends in antibiotic-resistant Acinetobacter spp.}

Both Carb-NS and MDR Acinetobacter spp. showed an overall significant linear decreasing trend in rates per 100 hospital admissions from 2013 to 2017 (Table 3, Fig. 2), with slopes of $-0.0009 /$ quarter and $-0.00134 /$ quarter, respectively (both $p<0.0001$ ) (Fig. 2a and c). The proportions of Carb-NS Acinetobacter spp. also showed an overall decreasing pattern, but the trend was insignificant $(p=0.2345)$ (Table 3 and Fig. 2b). Decreases in the proportions of MDR Acinetobacter spp. did achieve significance $(p<0.0001)$ (Table 3 and Fig. 2d). Both Carb-NS and MDR Acinetobacter spp. showed a strong seasonal changing pattern in the proportion of resistant isolates in which higher resistance rates were observed in the winter period (Q1) compared with the summer season (Q3) (Table 3 and Fig. $2 b$ and $d)$.

Table 2 Distribution of Pathogens by Culture Source

\begin{tabular}{|c|c|c|c|c|c|c|c|c|c|c|}
\hline \multirow[t]{3}{*}{ Source } & \multicolumn{6}{|l|}{ Enterobacteriaceae } & \multicolumn{4}{|c|}{ Acinetobacter spp. } \\
\hline & \multicolumn{2}{|l|}{$\overline{E S B L}$} & \multicolumn{2}{|l|}{ Carb-NS } & \multicolumn{2}{|l|}{ MDR } & \multicolumn{2}{|l|}{ Carb-NS } & \multicolumn{2}{|l|}{ MDR } \\
\hline & $\mathrm{n}$ (N Tested) & $\%$ & $\mathrm{n}$ (N Tested) & $\%$ & n (N Tested) & $\%$ & $\mathrm{n}$ (N Tested) & $\%$ & $\mathrm{n}$ (N Tested) & $\%$ \\
\hline All sources & $134,032(1,112,312)$ & $12.05 \%$ & $15,460(1,275,311)$ & $1.21 \%$ & $90,327(1,275,311)$ & $7.08 \%$ & $7243(19,325)$ & $37.48 \%$ & $9210(19,325)$ & $47.66 \%$ \\
\hline Urine & $89,224(775,699)$ & $11.50 \%$ & $7092(843,193)$ & $0.84 \%$ & $56,944(843,193)$ & $6.75 \%$ & $984(3201)$ & $30.74 \%$ & 1352 (3201) & $42.24 \%$ \\
\hline Skin/wound & $15,937(122,271)$ & $13.03 \%$ & $3174(167,125)$ & $1.90 \%$ & $11,852(167,125)$ & $7.09 \%$ & $2613(7024)$ & $37.20 \%$ & 3319 (7024) & $47.25 \%$ \\
\hline Respiratory & $10,325(59,316)$ & $17.41 \%$ & $3015(87,605)$ & $3.44 \%$ & $8959(87,605)$ & $10.23 \%$ & $2893(6429)$ & $45.00 \%$ & 3619 (6429) & $56.29 \%$ \\
\hline Blood & $13,654(105,998)$ & $12.88 \%$ & $1368(119,329)$ & $1.15 \%$ & $9167(119,329)$ & $7.68 \%$ & $532(1968)$ & $27.03 \%$ & 660 (1968) & $33.54 \%$ \\
\hline Other sources & $2895(24,343)$ & $11.89 \%$ & $450(29,505)$ & $1.53 \%$ & $2042(29,505)$ & $6.92 \%$ & $143(504)$ & $28.37 \%$ & $172(504)$ & $34.13 \%$ \\
\hline Intra-abdominal & $1997(24,685)$ & $8.09 \%$ & $361(28,554)$ & $1.26 \%$ & $1363(28,554)$ & $4.77 \%$ & 78 (199) & $39.20 \%$ & 88 (199) & $44.22 \%$ \\
\hline
\end{tabular}


Table 3 Model-detected Trends in Patterns of Resistance in Enterobacteriaceae and Acinetobacter spp. from 2013 to 2017

\begin{tabular}{|c|c|c|c|c|c|c|c|}
\hline \multirow[t]{2}{*}{ Pathogen Class } & \multirow[t]{2}{*}{ Pathogen } & \multirow[t]{2}{*}{ Measurement } & \multicolumn{3}{|c|}{ Overall (5-year) linear trend } & \multicolumn{2}{|l|}{ Seasonality } \\
\hline & & & Pattern & Quarterly slope $(95 \% \mathrm{Cl})$ & $p$ & Pattern & $p$ \\
\hline \multirow[t]{6}{*}{ Enterobacteriaceae } & ESBL & Rate per 100 admissions & Increasing & $0.0089(0.0052,0.0127)$ & $<0.0001$ & Insignificant & 0.0617 \\
\hline & & $\%$ of isolates tested & Increasing & $0.1510(0.0974,0.2045)$ & $<0.0001$ & Insignificant & 0.1168 \\
\hline & Carb-NS & Rate per 100 admissions & Increasing & $0.0004(0.0001,0.0006)$ & 0.0047 & Insignificant & 0.5075 \\
\hline & & $\%$ of isolates tested & Insignificant & $0.0013(-0.0027,0.0052)$ & 0.5331 & Insignificant & 0.1301 \\
\hline & MDR & Rate per 100 admissions & Decreasing & $-0.0022(-.0038,-.0006)$ & 0.0066 & Insignificant & 0.0501 \\
\hline & & $\%$ of isolates tested & Decreasing & $-0.0273(-0.0499,-0.0048)$ & 0.0176 & $\begin{array}{l}\text { Q1 higher, } \\
\text { Q3 lower }\end{array}$ & 0.0010 \\
\hline \multirow[t]{4}{*}{ Acinetobacter spp. } & Carb-NS & Rate per 100 admissions & Decreasing & $-0.0009(-0.0011,-0.0006)$ & $<0.0001$ & Insignificant & 0.1421 \\
\hline & & $\%$ of isolates tested & Insignificant & $-0.1075(-0.0904,0.0697)$ & 0.2345 & $\begin{array}{l}\text { Q1 higher, } \\
\text { Q3 lower }\end{array}$ & $<0.0001$ \\
\hline & MDR & Rate per 100 admissions & Decreasing & $-0.0014(-0.0017,-0.0010)$ & $<0.0001$ & Insignificant & 0.2189 \\
\hline & & $\%$ of isolates tested & Decreasing & $-0.4269(-0.5793,-0.2744)$ & $<0.0001$ & $\begin{array}{l}\text { Q1 higher, } \\
\text { Q3 lower }\end{array}$ & $<0.0001$ \\
\hline
\end{tabular}

Abbreviations: Carb-NS carbapenem-nonsusceptible, Cl confidence interval, ESBL extended-spectrum beta-lactamase-producing, MDR multidrug resistant, Q quarter

\section{Discussion}

Antimicrobial resistance in Gram-negative pathogens continues to threaten public health and increase the societal cost of health care. Carbapenem-resistant pathogens are a particular problem due to limited treatment options, ability to infect multiple organ systems, and high attributable mortality and cost [13, 20]. Although CRE are unquestionably an important clinical concern, ESBL and MDR Enterobacteriaceae pose a substantial threat to patient health and are present in greater numbers $[1,6]$. Based on our Q4 2017 data, these pathogens accounted for a 10-fold higher incidence of pathogens in hospitalized patients than Carb-NS Enterobacteriaceae. Acinetobacter spp., a rare but dangerous Gram-negative pathogen, is associated with high rates of resistance and mortality $[7,21]$.

Information on trends in susceptibility is important from public health, infection prevention, and antimicrobial development standpoints. Some recent reports suggest that rates of antimicrobial-resistant Enterobacteriaceae are decreasing, including an NHSN study of central line-associated BSIs and catheter-associate UTIs [8]. However, other studies suggest a continued increase in the rate of antimicrobial-resistant Gram-negative pathogens [22-24]. Outside of hospitals, there has been an increase in antibiotic-resistant Gram-negative pathogens in poultry and livestock [25], which may contribute to the spread of infections in the community.

Our study evaluated the rates of antimicrobial-resistant or nonsusceptible Enterobacteriaceae and Acinetobacter spp. from multiple culture sources in hospitalized patients throughout the United States from 2013 to 2017. We found that rates of ESBL and Carb-NS Enterobacteriaceae per 100 hospital admissions increased significantly during this time period, although increases were fairly modest, while rates of MDR Enterobacteriaceae, MDR Acinetobacter spp., and Carb-NS Acinetobacter spp. decreased. Analyses of the proportions of resistant isolates largely mirrored the trends observed with rates per 100 hospital admission, although increases in the proportion of Carb-NS Enterobacteriaceae and decreases in the proportion of Carb-NS Acinetobacter spp. did not reach statistical significance. For Acinetobacter spp., decreases were fairly constant throughout the 5-year period. In contrast, MDR Enterobacteriaceae rates and proportion of resistant isolates initially increased followed by a decrease from Q2 2015 to Q4 2017. Although this recent trend is encouraging, decreases in MDR Enterobacteriaceae were modest and rates are still high (6.8\% in Q4 2017).

Independent reports of antimicrobial resistance and non-susceptibility are difficult to compare because they are influenced by a number of factors, including number of participating institutions, culture site(s), pathogens evaluated, definitions of resistance, culture ordering practices, and pathogen sources (community- versus hospital-acquired or surveillance versus infection). There are thus several reasons why our findings of increases in ESBL and Carb-NS Enterobacteriaceae might differ from studies reporting decreased rates of antimicrobial-resistant Enterobacteriaceae. Most notable is that our analyses include cultures collected in the admission period as well as those cultured in the hospital-onset time period; our analyses were confined to hospitalized patients, but were not specific to hospital-acquired infections. In contrast, CDC reports of hospital-acquired infections, by definition, attempt to exclude patients admitted with infections. In many facilities, hospital-acquired infections are targeted for intensive infection control and antimicrobial stewardship efforts, which may result in 


\section{Rate per 100 Admissions}

A

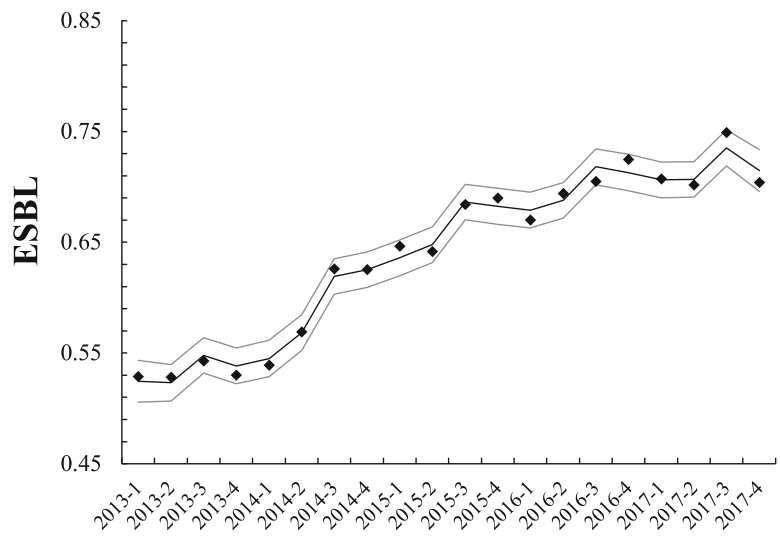

C

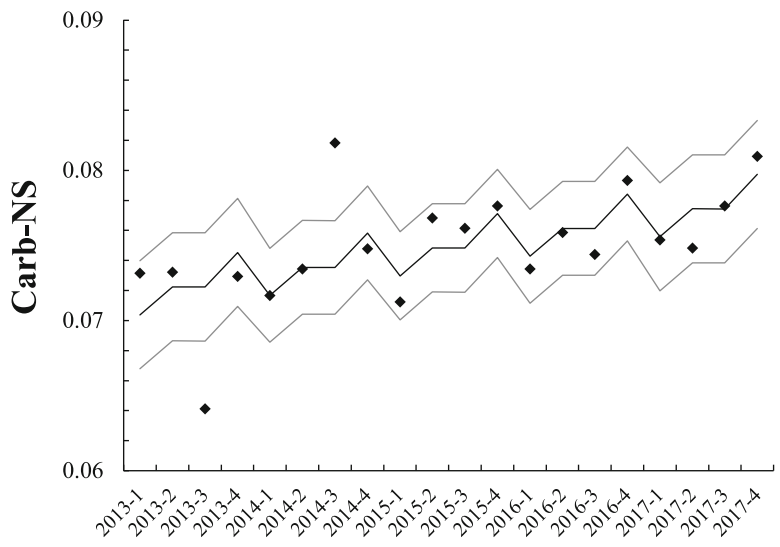

E

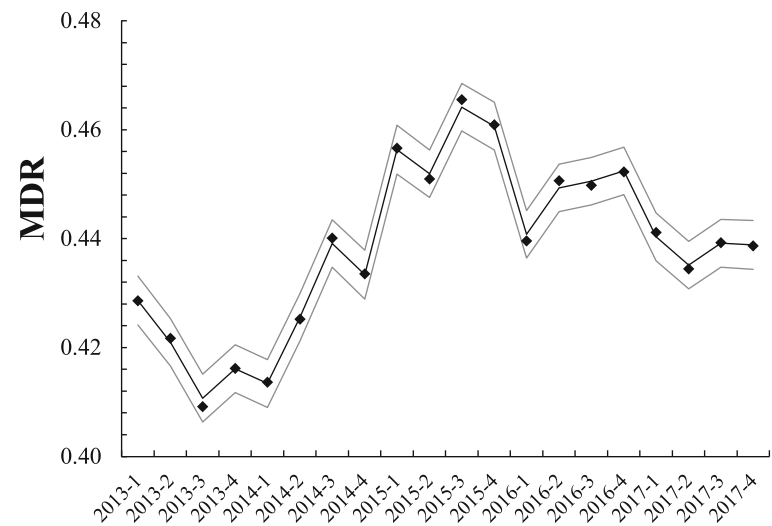

\% Resistant Isolates

B

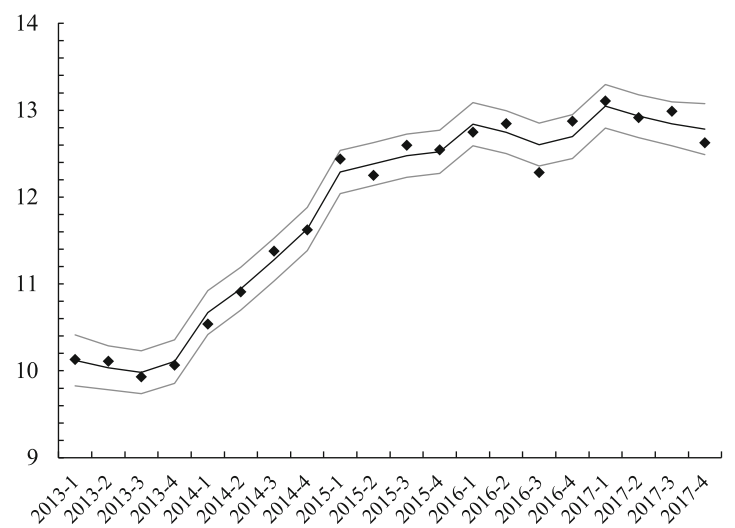

D

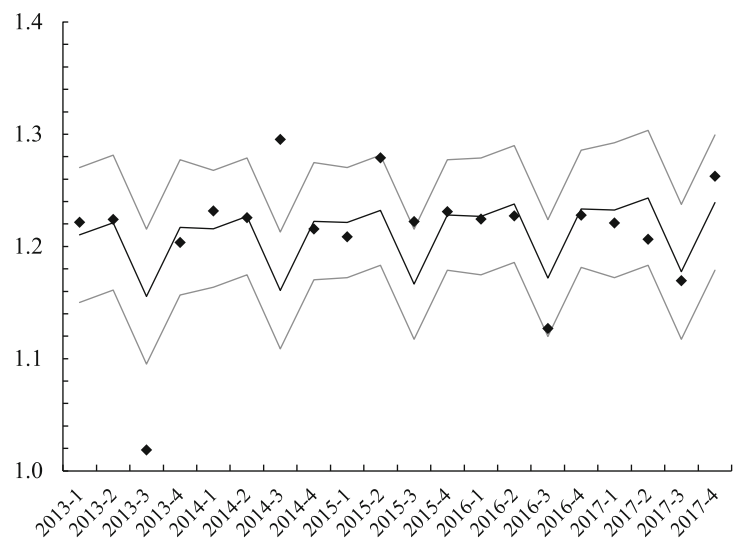

F

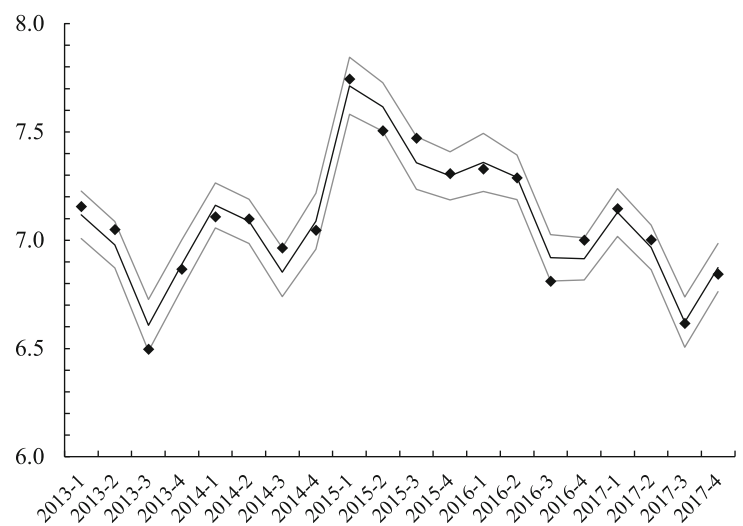

Fig. 1 Observed and model-estimated resistance trends in antibiotic-resistant Enterobacteriaceae by year-quarter. a Extended-spectrum betalactamase (ESBL)-producing Enterobacteriaceae per 100 hospital admissions and (b) as a proportion of tested isolates; (c) carbapenemnonsusceptible (Carb-NS) Enterobacteriaceae per 100 hospital admissions and (d) as a proportion of tested isolates; and (e) multidrug-resistant (MDR) Enterobacteriaceae per 100 hospital admissions and ( $\mathbf{f}$ ) as a proportion of tested isolates; and. Diamonds indicate observed data, solid lines indicate predicted trends, and dotted lines indicate $95 \%$ confidence intervals 


\section{Rate per 100 Admissions}

A

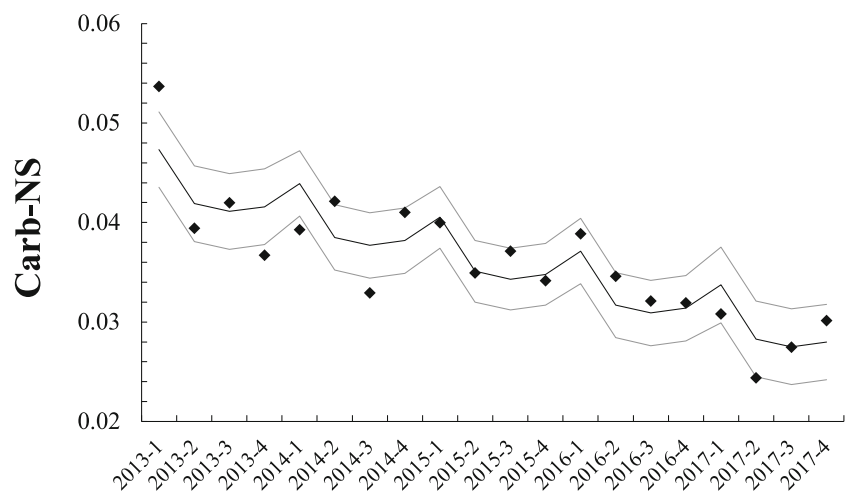

C

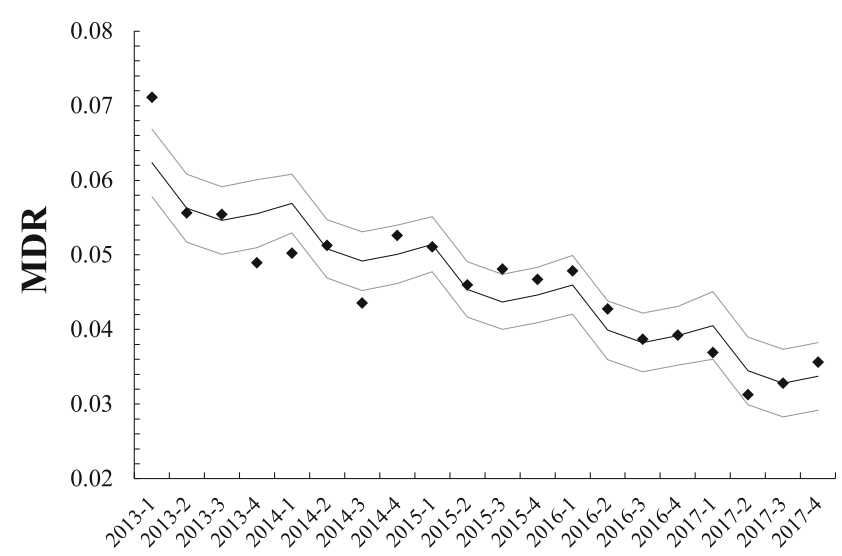

\% Resistant Isolates

B

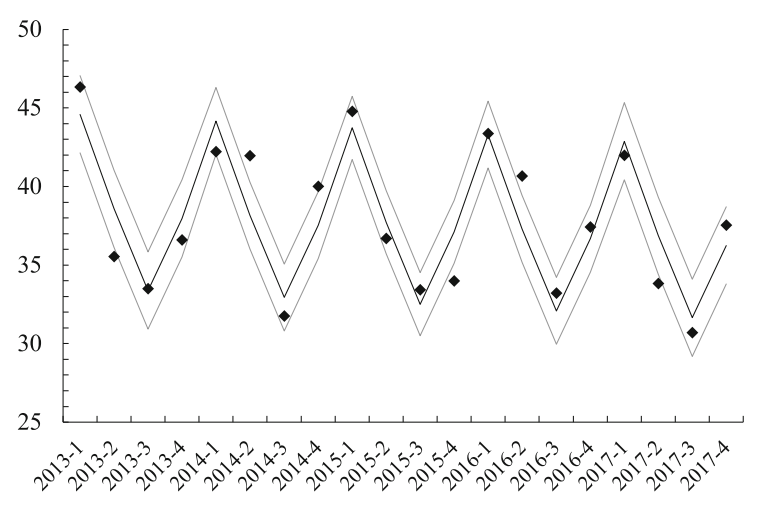

D

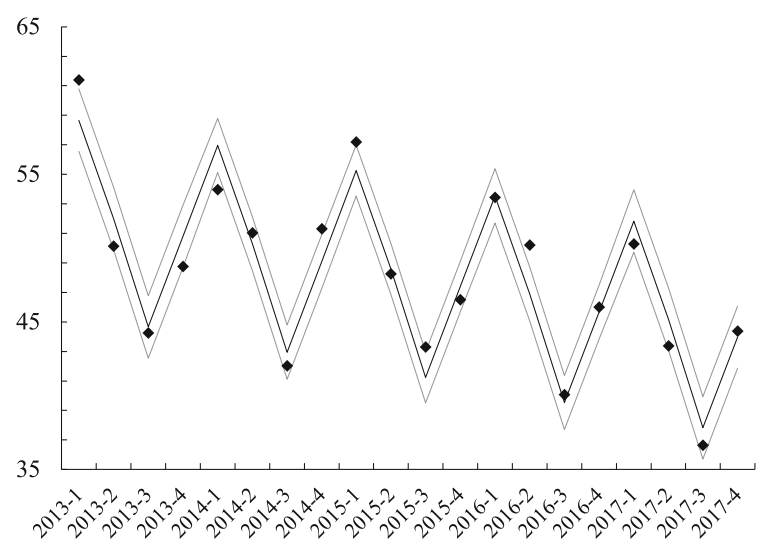

Fig. 2 Observed and model-estimated resistance trends in Acinetobacter spp. by year-quarter. a Carbapenem-nonsusceptible (Carb-NS) Acinetobacter spp. per 100 hospital admissions and (b) as a proportion of tested isolates; (c) multidrug-resistant (MDR) Acinetobacter spp. per 100 hospital admissions and (d) as a proportion of tested isolates;. Diamonds indicate observed data, solid lines indicate predicted trends, and dotted lines indicate 95\% confidence intervals

decreased antibiotic resistance in these infections compared with the resistance profile of pathogens in the community at large. The geographic representation of included hospitals can also influence results. We have shown previously that there are significant differences in rates of antibiotic-resistant Acinetobacter spp. and MDR Enterobacteriaceae across geographic regions in the US [12].

Our results concerning recent decreases in antibiotic-resistant Acinetobacter infections are consistent with other observations [6, 26]. Others have also observed seasonal variations in US Acinetobacter infections [27, 28], but previous studies have reported increased infection rates in summer months compared with winter months, whereas we found increased proportions of resistant pathogens (but not rates per 100 admissions) in the winter months.
We observed a similar pattern for MDR Enterobacteriaceae, consistent with a report on antibiotic-resistant E. coli in which higher proportions of resistant isolates in winter months correlated with antibiotic prescribing practices [29]. The reasons for discrepancy in seasonal trends among different studies may involve differences in study methodology, in particular the inclusion of admission period cultures rather than solely "hospital onset" isolates and assessment of the proportion of resistant isolates versus overall frequencies of infection.

Limitations of our study include the collection and analysis of data from non-duplicated unique collected cultures rather than from unique patients. We are therefore unable to evaluate clinical outcomes associated with the antibiotic-resistant pathogens in this study. The results reported here represent non-duplicate culture 
positive isolates and not confirmed invasive infections. Our study included only selected Acinetobacter spp., and mechanisms of resistance were not investigated. Susceptibility was based on local microbiology practices at each facility and not standardized across facilities. Enterobacteriaceae ESBL and CRE testing practices and breakpoints are known to vary among different institutions [30], and institutions with lower breakpoints typically report higher resistance rates [31]. In particular, delayed implementation of updated Clinical \& Laboratory Standards Institute guidelines for third-generation cephalosporins, cefepime, and carbapenem breakpoints may have influenced susceptibility assessments. These breakpoints were lowered in 2010 (carbapenems and third-generation cephalosporins) and 2014 (cefepime) for Enterbacteriaceae and in 2014 (carbapenems) for Acinetobacter spp., but immediate adoption in hospital laboratories likely varied due to the use of automated antimicrobial susceptibility testing systems. In one study of 25 US community hospitals, only 5 (20\%) had adopted the 2010 carbapenem breakpoints by the end of 2012 [31]. It is possible that the increased resistance observed in ESBL and Carb-NS Enterobacteriaceae in our study may reflect the expanding adoption of these lower breakpoints, leading to the identification of a greater number of isolates as antibiotic resistant. The inclusion of isolates with intermediate resistance increased the number of isolates slightly, so our data of nonsusceptible isolates cannot be strictly compared to studies of pathogens meeting criteria for resistance. Antimicrobial susceptibility results for ertapenem may have also influenced Carb-NS rates, as AmpC hyperproduction and porin changes can cause some isolates to be Carb-NS to ertapenem while remaining susceptible to other carbapenems [32]. Despite these limitations, we believe our study provides an epidemiological lens on trends in antimicrobial resistance that reflects both the community and inpatient burden of hospitalized patients and may help inform further studies.

Our findings highlight the multiple factors influencing infections with resistant pathogens. Antibiotic-resistant Gram-negative bacteria are found in multiple culture sources, often spread from non-infected fomites and contacts, and can be ubiquitously represented in intensive care, medical, and surgical hospital wards [33]. Although understandably a focus of attention, reportable healthcareacquired infections represent only a portion of clinically relevant infections. The inclusion of multiple sources of positive cultures may represent a more accurate risk of spreading drug-resistant organisms between patients, healthcare workers and family members [34, 35] or from community food supplies [25]. Additional Gram-negative bacteria, particularly Pseudomonas aeruginosa, also pose important clinical challenges. Work is in progress to explore trends in antibiotic-resistant $P$. aeruginosa.

\section{Conclusion}

Our findings from this large-scale study of patients in US hospitals show increasing numbers of infections due to ESBL and Carb-NS Enterobacteriaceae between 2013 and 2017. These data support continuing efforts by the $\mathrm{CDC}$ and WHO to combat these pathogens. Infections caused by MDR and Carb-NS Acinetobacter spp. are decreasing, but Acinetobacter remains a dangerous and difficult-to-treat pathogen. Continued infection control efforts, together with diagnostic and antimicrobial stewardship and new antibiotics to expand treatment options, will be required to manage these antibioticresistant Gram-negative pathogens.

\section{Additional files}

Additional file 1: Specific antibiotics for multidrug resistance. (DOCX $18 \mathrm{~kb}$ )

Additional file 2: Descriptive statistics of resistance in

Enterobacteriaceae over time. Rate indicates resistance per 100

admissions and \% indicates proportion of resistant isolates (resistant isolates/total isolates tested). (DOCX $23 \mathrm{~kb}$ )

Additional file 3: Descriptive statistics of resistance in Acinetobacter spp. over time. Rate indicates resistance per 100 admissions and \% indicates proportion of resistant isolates (resistant isolates/total isolates tested). (DOCX $22 \mathrm{~kb}$ )

\section{Abbreviations}

ARIMA: Autoregressive integrated moving average; BD: Becton, Dickinson and Company; BSI: Bloodstream infection; Carb-NS: Carbapenem-nonsusceptible; CDC: Centers for Disease Control and Prevention; CRE: Carbapenem-resistant Enterobacteriaceae; ESBL: Extended-spectrum beta-lactamase; GEE: Generalized estimating equation; GLMM: General linear mixed model; MDR: Multidrug resistant; NHSN: US National Healthcare Safety Network; Q: Quarter; SAS: Statistical Analysis System; UCM: Unobserved component model; UTI: Urinary tract infection; WHO: World Health Organization

\section{Acknowledgments}

The authors thank James Baggs, Sarah Yi, and Kelly Hatfield from the CDC Division of Healthcare Quality Promotion for their review and guidance on this manuscript, and Sharon L. Cross, Ph.D. (Fusion MD Medical Science Network, Inc., Montreal, Canada) for assistance in preparing and editing the manuscript with funding from Becton, Dickinson \& Company.

\section{Authors' contributions}

VG and GY drafted the manuscript and GY conducted statistical analyses. VG, $\mathrm{GY}, \mathrm{MO}$, and $\mathrm{KY}$ developed the study concept and design. VG, GY, MO, KL, $J M$, and $K Y$ were involved in data analysis and interpretation and read and approved the final manuscript.

\section{Funding}

This work was supported by Tetraphase Pharmaceuticals. Tetraphase was involved in study design, interpretation of data, and approval of the manuscript for submission. Medical writing support was provided by Becton, Dickinson \& Company.

\section{Availability of data and materials}

The datasets used and/or analysed during the current study are available from the corresponding author on reasonable request.

\section{Ethics approval and consent to participate}

Outcome studies using this retrospective, deidentified dataset were approved and informed consent was waived by the New England Institutional Review Board (Wellesley, Massachusetts). 


\section{Consent for publication}

Not applicable.

\section{Competing interests}

VG, GY, JM, and KY are employees of Becton, Dickinson \& Company, Franklin Lakes, New Jersey. MO and KL are employees of Tetraphase Pharmaceuticals, Watertown, Massachusetts.

Received: 10 April 2019 Accepted: 15 August 2019

Published online: 23 August 2019

\section{References}

1. Centers for Disease Control and Prevention. Antibiotic resistance threats in the United States, 2013. 2013. https://www.cdc.gov/drugresistance/threatreport-2013/pdf/ar-threats-2013-508.pdf. Accessed 6 Aug 2018.

2. World Health Organization. Global priority list of antibiotic-resistant bacteria to guide research, discover, and development of new antibiotics. 2017. http://apps.who.int/medicinedocs/documents/s23171en/s23171en.pdf. Accessed 6 Aug 2018.

3. Tacconelli E, Carrara E, Savoldi A, Harbarth S, Mendelson M, Monnet DL, et al. Discovery, research, and development of new antibiotics: the WHO priority list of antibiotic-resistant bacteria and tuberculosis. Lancet Infect Dis. 2018;18:318-27.

4. Zalacain M, Biedenbach DJ, Badal RE, Young K, Motyl M, Sahm DE, Pathogen prevalence and antimicrobial susceptibility among Enterobacteriaceae causing hospital-associated intra-abdominal infections in adults in the United States (2012-2013). Clin Ther. 2016;38:1510-21.

5. Zilberberg MD, Nathanson BH, Sulham K, Fan W, Shorr AF. Carbapenem resistance, inappropriate empiric treatment and outcomes among patients hospitalized with Enterobacteriaceae urinary tract infection, pneumonia and sepsis. BMC Infect Dis. 2017;17:279.

6. Weiner LM, Webb AK, Limbago B, Dudeck MA, Patel J, Kallen AJ, et al. Antimicrobial-resistant pathogens associated with healthcare-associated infections: summary of data reported to the National Healthcare Safety Network at the Centers for Disease Control and Prevention, 2011-2014. Infect Control Hosp Epidemiol. 2016;37:1288-301.

7. Zilberberg MD, Kollef MH, Shorr AF. Secular trends in Acinetobacter baumannii resistance in respiratory and blood stream specimens in the United States, 2003 to 2012: a survey study. J Hosp Med. 2016;11:21-6.

8. Woodworth KR, Walters MS, Weiner LM, Edwards J, Brown AC, Huang JY, et al. Vital signs: containment of novel multidrug-resistant organisms and resistance mechanisms - United States, 2006-2017. MMWR Morb Mortal Wkly Rep. 2018;67:396-401.

9. Brossette SE, Hacek DM, Gavin PJ, Kamdar MA, Gadbois KD, Fisher AG, et al. A laboratory-based, hospital-wide, electronic marker for nosocomial infection: the future of infection control surveillance? Am J Clin Pathol. 2006:125:34-9.

10. Ridgway JP, Sun X, Tabak YP, Johannes RS, Robicsek A. Performance characteristics and associated outcomes for an automated surveillance tool for bloodstream infection. Am J Infect Control. 2016;44:567-71.

11. Tabak YP, Zilberberg MD, Johannes RS, Sun X, McDonald LC. Attributable burden of hospital-onset Clostridium difficile infection: a propensity score matching study. Infect Control Hosp Epidemiol. 2013;34:588-96.

12. Gupta V, Ye G, Olesky M, Lawrence K, Murray J, Yu K. National prevalence estimates for resistant Enterobacteriaceae and Acinetobacter species in hospitalized patients in the United States. Int J Infect Dis. 2019;85:203-11.

13. McCann E, Srinivasan A, DeRyke CA, DePestel DD, Murray J, Gupta V. Carbapenem-nonsusceptible Gram-negative pathogens in ICU and non-ICU settings in US hospitals in 2017: a multicenter study. Open Forum Infect Dis. 2018;5:ofy241.

14. Magiorakos A-P, Srinivasan A, Carey RB, Carmeli Y, Falagas ME, Giske CG, et al. Multidrug-resistant, extensively drug-resistant and pandrug-resistant bacteria: an international expert proposal for interim standard definitions for acquired resistance. Clin Microbiol Infect. 2012;18:268-81.

15. Centers for Disease Control and Prevention. Facility guidance for control of carbapenem-resistant Enterobacteriaceae (CRE). 2015. https://www.cdc.gov/ hai/pdfs/cre/CRE-guidance-508.pdf. Accessed 8 Aug 2018.

16. Centers for Disease Control and Prevention. Antimicrobial use and resistance (AUR). 2018. https://www.cdc.gov/nhsn/pdfs/pscmanual/11 pscaurcurrent.pdf. Accessed 6 Aug 2018.
17. Centers for Disease Control and Prevention. Multidrug-resistant organisms and Clostridium difficile infection (MDRO/CDI) module. 2018. https://www.cdc.gov/ nhsn/pdfs/pscmanual/12pscmdro_cdadcurrent.pdf. Accessed 6 Aug 2018.

18. Harvey AC. Forecasting, structural time series models and the Kalman filter. Cambridge: Cambridge University Press; 1989.

19. Harvey A, Koopman SJ. Messy time series: a unified approach. In: Fomby TB, Hill RC, editors. Advances in econometrics: messy data-missing observations, outliers, and mixed-frequency data, vol. 13. Bingley: Emerald Group Publishing Limited; 2015. p. 103-43.

20. Bartsch SM, McKinnell JA, Mueller LE, Miller LG, Gohil SK, Huang SS, et al. Potential economic burden of carbapenem-resistant Enterobacteriaceae (CRE) in the United States. Clin Microbiol Infect. 2017;23:48-e9-48-e.16.

21. Lemos EV, de la Hoz FG, Einarson TR, McGhan WF, Quevedo E, Castaneda C, et al. Carbapenem resistance and mortality in patients with Acinetobacter baumannii infection: systematic review and meta-analysis. Clin Microbiol Infect. 2014;20:416-23.

22. McDanel J, Schweizer M, Crabb V, Nelson R, Samore M, Khader K, et al. Incidence of extended-spectrum $\beta$-lactamases (ESBL)-producing Escherichia coli and Klebsiella infections in the United States: a systematic literature review. Infect Control Hosp Epidemiol. 2017;38:1209-15.

23. Thaden JT, Fowler VG, Sexton DJ, Anderson DJ. Increasing incidence of extended-spectrum $\beta$-lactamase-producing Escherichia coli in community hospitals throughout the southeastern United States. Infect Control Hosp Epidemiol. 2016;37:49-54.

24. Hackel MA, Badal RE, Bouchillon SK, Biedenbach DJ, Hoban DJ. Resistance rates of intra-abdominal isolates from intensive care units and non-intensive care units in the United States: The Study for Monitoring Antimicrobial Resistance Trends 2010-2012. Surg Infect (Larchmt). 2015;16:298-304.

25. Food and Drug Administration. National Antimicrobial Resistance Monitoring System 2014 Trend Analysis 2014. https://www.fda.gov/ downloads/AnimalVeterinary/SafetyHealth/AntimicrobialResistance/ NationalAntimicrobialResistanceMonitoringSystem/UCM529799.pdf. Accessed 4 Sept 2018.

26. Russell DL, Uslan DZ, Rubin ZA, Grogan TR, Martin EM. Multidrug resistant Acinetobacter baumannii: a 15-year trend analysis. Infect Control Hosp Epidemiol. 2018;39:608-11.

27. Perencevich EN, McGregor JC, Shardell M, Furuno JP, Harris AD, Morris JG Jr. et al. Summer peaks in the incidences of gram-negative bacterial infection among hospitalized patients. Infect Control Hosp Epidemiol. 2008;29:1124-31.

28. Eber MR, Shardell M, Schweizer ML, Laxminarayan R, Perencevich EN. Seasonal and temperature-associated increases in gram-negative bacterial bloodstream infections among hospitalized patients. PLoS One. 2011;6:e25298.

29. Sun L, Klein EY, Laxminarayan R. Seasonality and temporal correlation between community antibiotic use and resistance in the United States. Clin Infect Dis. 2012;55:687-94.

30. Shugart A, Walters MS, Weiner LM, Lonsway D, Kallen AJ. Hospital microbiology laboratory practices for Enterobacteriaceae: Centers for Disease Control and Prevention National Healthcare Safety Network (NHSN) annual survey, 2015 and 2016. Infect Control Hosp Epidemiol. 2018;39:1115-7.

31. Thaden JT, Lewis SS, Hazen KC, Huslage K, Fowler VG Jr, et al. Rising rates of carbapenem-resistant enterobacteriaceae in community hospitals: a mixedmethods review of epidemiology and microbiology practices in a network of community hospitals in the southeastern United States. Infect Control Hosp Epidemiol. 2014:35:987-3.

32. Birgy $A$, Bidet $P$, Genel $N$, Doit $C$, Decré $D$, Arlet $G$, et al. Phenotypic screening of carbapenemases and associated $\beta$-lactamases in carbapenemresistant Enterobacteriaceae. J Clin Microbiol. 2012;50:1295-302.

33. Peleg AY, Hooper DC. Hospital-acquired infections due to gram-negative bacteria. N Engl J Med. 2010:362:1804-13.

34. Eveillard M, Martin Y, Hidir N, Boussougant Y, Joly-Guilou ML. Carriage of methicillin-resistant Staphylococcus aureus among hospital employees: prevalence, duration, and transmission to households. Infect Control Hosp Epidemiol. 2004;25:114-20.

35. Miller L, Eells SJ, David MZ, Ortiz N, Taylor AR, Kumar N, et al. Staphylococcus aureus skin infection recurrences among household members: an examination of host, behavioral, and pathogen-level predictors. Clin Infect Dis. 2015;60:753-63.

\section{Publisher's Note}

Springer Nature remains neutral with regard to jurisdictional claims in published maps and institutional affiliations. 